\title{
Severe Obstructive Sleep Apnea Managed with Mandibular Advancement Device in Pediatrics: An Interdisciplinary Case Report
}

\author{
Devon M. Ptak, MPH, Ruba Alkadhi, BDS, MS, II Ho Tae, DDS, MS, PhD, Leopoldo P. Correa, BDS, MS
}

Tufts University School of Dental Medicine, Boston, MA

\begin{abstract}
Mandibular advancement devices (MADs) may enhance upper airway patency during sleep by enlarging the upper airway and/or by decreasing upper airway collapsibility, thereby helping to prevent obstruction of the upper airway. ${ }^{1}$ Although MADs are commonly used for the management of obstructive sleep apnea (OSA) in adults, their use in pediatric patients is less common, with limited information available regarding their efficacy. ${ }^{2}$ However, for pediatric patients in whom continuous positive airway pressure (CPAP) has failed, who are interested in nonsurgical interventions, or who are awaiting future surgical interventions, an MAD may be an option. This report details one such case, and the successful treatment of OSA with an MAD after failure to tolerate CPAP in a pediatric patient awaiting orthognathic surgery.
\end{abstract}

Keywords: Pediatric OSA; Mandibular Advancement Device; Severe Obstructive Sleep Apnea; Interdisciplinary Management Citation: Ptak D, Alkadhi R, Tae IH, Correa LP. Severe Obstructive Sleep Apnea Managed with Mandibular Advancement Device in Pediatrics: An Interdisciplinary Case Report. J Dent Sleep Med. 2019;6(2)

\section{INTRODUCTION}

The prevalence of obstructive sleep apnea (OSA) in childhood is approximately $3 \% .^{3}$ Appropriate management of OSA is determined on an individual basis, with adenotonsillectomy recommended as the first line of treatment of patients with adenotonsillar hypertrophy. ${ }^{4}$ Continuous positive airway pressure (CPAP) is considered a safe and effective approach for patients in whom surgical intervention is not indicated or who have preference for a nonsurgical approach. ${ }^{5}$ There is limited literature on the management of pediatric OSA with a mandibular advancement device (MAD) in cases where the patient is awaiting orthognathic surgery; however, the available literature does suggest that MAD therapy may result in improvement in apnea-hypopnea index (AHI) scores for pediatric patients with OSA. ${ }^{6}$ This case report highlights the use of an MAD in a pediatric patient, after failure to tolerate CPAP, and prior to scheduled orthognathic surgery.

\section{REPORT OF CASE}

A 14-year-old boy presented with severe OSA (AHI= 52/h). A diagnostic test was performed using a level I sleep test (polysomnography [PSG]) and the results were reviewed and interpreted by a board-certified sleep physician. The patient's chief complaint was excessive daytime sleepiness, frequent nighttime awakenings, and headaches. He experienced snoring during infancy and underwent tonsillectomy and adenoidectomy at age 4 years.

His medical conditions included: macrocephaly, neuroma of foot, jaw asymmetry, mandibular hypoplasia, hypoplastic left condyle, and allergic rhinitis. The patient received a diagnosis of skeletal II malocclusion and mandibular hypoplasia by the oral and maxillofacial surgeon.

At the time of clinical examination, the patient's height was 5 feet 9 inches, weight was $160 \mathrm{lb}$, and body mass index was $23.6 \mathrm{~kg} / \mathrm{m}^{2}$. His neck circumference was 14.5 inches and Friedman tongue position was III with scalloping around the lateral border of tongue. The patient's soft palate was elongated and the uvula was of medium size. His mandibular midline was shifted to the right side by $2 \mathrm{~mm}$. Temporomandibular joint examination revealed no joint sound and no limitation of opening with deflection to the left side. The patient showed moderate tenderness of the bilateral lateral pterygoid muscle, moderate tenderness of the left masseteric muscle, moderate tenderness of the bilateral temporal muscle, moderate tenderness of the left sternocleidomastoid muscle, and moderate tenderness of the bilateral posterior neck and shoulder muscles.

Baseline diagnostic PSG showed severe OSA with AHI of 52/h, with lowest arterial oxygen saturation (LSAT) of $78 \%$. Positive airway pressure therapy was prescribed but patient intolerance to the machine resulted in discontinuation. The patient was referred for consultation with the oral and maxillofacial surgeon for correction of mandibular hypoplasia and jaw asymmetry. The oral and maxillofacial surgeon recommended MAD therapy as a 
transitional therapy until the patient is ready for orthognathic surgery after the growth cessation.

Bite registration was taken at $80 \%$ of maximum jaw protrusion, and a custom-fabricated and adjustable MAD was fitted. Patient accommodation to the appliance was successful, with good adherence and without discomfort. Follow-up level I sleep study (PSG), implemented and interpreted by a board-certified sleep physician, with MAD showed complete resolution of sleep apnea with AHI of 0.9 and LSAT of 93\% (Figure 1). Patient-reported daytime sleepiness as measured by Epworth Sleepiness Scale (ESS) was 7 at baseline and reduced to 1 with MAD therapy. The patient reported a decrease in the frequency and intensity of headaches.

\section{DISCUSSION}

There is limited literature on the management of pediatric OSA with MADs, and the use of MADs is not typically included in guidelines for the treatment of OSA in pediatric patients. However, the successful change in AHI, ESS, and patient adherence demonstrated in this case report support the idea that MADs may be a viable treatment option for selected pediatric patients with OSA who are nonresponsive to traditional treatment options, or as an interim appliance for those awaiting surgical intervention.

\section{REFERENCES}

1. Villa MP, Miano S, Rizzoli A. Mandibular advancement devices are an alternative and valid treatment for pediatric obstructive sleep apnea syndrome. Sleep Breath. 2012;16(4):971-976.

2. Idris G, Galland B, Robertson CJ, Farella M. Efficacy of a mandibular advancement appliance on sleep disordered breathing in children: a study protocol of a crossover randomized controlled trial. Front Physiol. 2016;7:353.

3. Chang SJ, Chae KY. Obstructive sleep apnea syndrome in children: epidemiology, pathophysiology, diagnosis and sequelae. Korean $J$ Pediatr. 2010 Oct;53(10):863-871.

4. Marcus CL, Brooks LJ, Draper KA, et al. Diagnosis and management of childhood obstructive sleep apnea syndrome. Pediatrics. 2012;130(3):576-584

5. Marcus CL, Ward SL, Mallory GB, et al. Use of nasal continuous positive airway pressure as treatment of childhood obstructive sleep apnea. J Pediatr. 1995 Jul;127(1):88-94.

6. Nazarali N, Altalibi M, Nazarali S, Major MP, Flores-Mir C, Major PW. Mandibular advancement appliances for the treatment of paediatric obstructive sleep apnea: a systematic review. Eur J Orthod. 2015;37(6):618-626.

\section{SUBMISSION \& CORRESPONDENCE INFORMATION}

\section{Submitted for publication May 29, 2018 \\ Submitted in final revised form October 26, 2018 \\ Accepted for publication January 22, 2019}

Address correspondence to: Leopoldo Correa, BDS, MS, Tufts University School of Dental Medicine, Boston, MA, Email: Leopoldo.Correa@tufts.edu

\section{DISCLOSURE STATEMENT}

The authors report no conflicts of interest. 


\section{Figure 1 - Mandibular Advancement Device and Apnea-Hypopnea Index}

A.

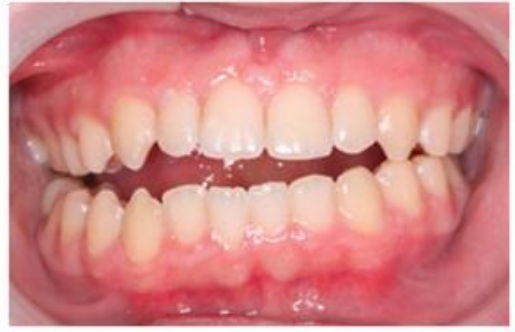

B.

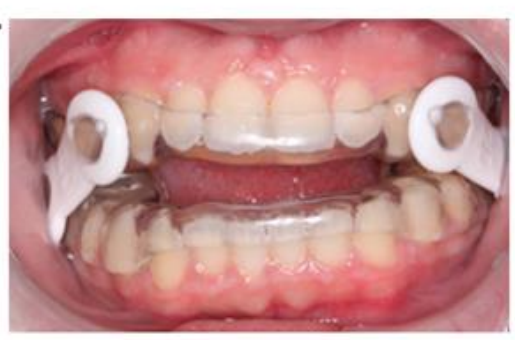

\section{AHI Pre and Post MAD Treatment}

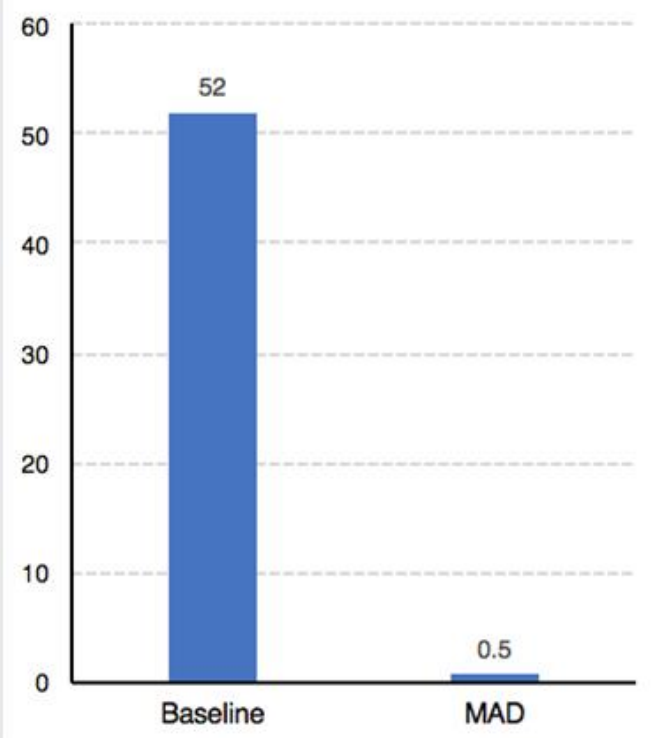

A, B: Intraoral view of patient with and without MAD. C: Effects of the Mandibular Advancement Device (MAD) on apnea-hypopnea index (AHI). 